\title{
Trust in Food and Trust in Science
}

\author{
Matthias Kaiser $^{1} \cdot$ Anne Algers ${ }^{2}$
}

Published online: 18 August 2017

(C) Springer International Publishing AG 2017

Recent and dramatic knowledge depreciation has raised the need for science to take an active role in the public debate and widen the reference frames. Publishing appealing narratives introducing a common reference frame may provide positive identification stimuli. The traditional voice of science has - rightly or wrongly - been conceived and portrayed as dry and lifeless, not capable of guiding our policies towards a future we want. In times when even simple facts are questioned, and society is more and more dependent on scientific rigor underpinning its policy decisions, academics have high responsibilities. The flip-side of responsibility is trust. If trust in us as individual scientists or in science as a social institution is eroded, then why take on responsibility?

In regard to the topics of this journal, ethical issues of trust point us at least in two directions: one in the direction of the food we eat, as trust in the good qualities of this food; the other points us to those who know something more about this food than most people, the scientists who study, develop and deal with food. Trust seems such an innocent term, but as soon as we look into the matter a bit deeper, far reaching implications occur (Meijboom 2008). We all know that trust is easily lost, but hard to re-gain. What triggers trust? What qualities convey trust and what features reduce trust?

We do not have answers to all these important questions, but we believe that this issue of Food Ethics throws light on various aspects connected to trust.

The paper" How to Label 'Natural' Foods: A matter of Complexity" by Sandin discusses the complexity of the concept of naturalness. This opens up for different interpretations by the producer or manufacturer and the consumer which may give rise to tension. Furthermore, it suggests labelling to reflect the complexity of any food item.

Matthias Kaiser

matthias.kaiser@uib.no

Anne Algers

anne.algers@gu.se

1 Organization Department of Education, Communication and Learning, Gothenburg University, P.O. Box 300, Gothenburg, Sweden

2 Department of Applied Information Technology, Gothenburg University, Gothenburg, Sweden 
Naturalness and trust are also the themes for the paper "Perceptions of Ethical Challenges within the LowInputBreeds Project" written by Jensen, Michalopoulos, Meijboom and Gjerris.

Trust in labelling is the concern of the paper" Halal meat fraud and safety issues in the UK: a review in the context of the European Union" by Fuseini, Wotton, Knowles and Hadley.

This paper focuses on fraud related to the mislabelling of halal meat and is based on a case from the UK where pork meat was found in some processed "Halal" products.

For those following the laws of their religion trust in the market is essential. However, when quality assurance and certification organizations fail to capture the problem with fraudulent activity, the issue goes beyond religion and culture. The paper relates to the article by Miele published in the last issue; both are bringing up the issue of Halal rules and concerns for animal welfare related to slaughter without prior stunning of the animal.

The tensions between producers and consumers of food production are aggravated by the fact that "more than half of the poorest people in the world currently achieve their food capabilities through a direct production entitlement" while the needs of the urban poor is not recognized (Thompson 2015, p. 120). This is in turn the topic of the paper "A capability approach to food choices" by Gombert, Douglas, McArdle and Carlisle. Obviously, there are no easy answers and the association made between health and capabilities by others (e.g. Ruger 2003), views health as the combination of the influence of socioeconomic structures, as well as personal agency, a view which many may regard as at best incomplete.

Many times this trust in the food is anchored in the ways it is produced or by whom it is produced. But it may also relate directly to the stuff which is eaten. A good example is the discussion about eating insects. Can it really be good and / or safe to eat organisms which we oftentimes detest having around us? The paper by Waltner-Toews and Houle is zooming in on eating insects for sustainability and food security reasons. It raises the issue, but when it comes to the ethical questions the authors feel all we can do is start a conversation about it - and that is what they do.

And then there is trust in the experts about food. Some experts appear very confident in their ability to make reliable predictions about the ongoing and future developments in regard food. But is their reasoning good enough? Saltelli and Lo Piano ask this question in "Problematic quantifications: a critical appraisal of scenario making for a global 'sustainable' food production". Following their criticism would arguably tend to diminish the trust in at least some of the self-acclaimed experts.

When we pay our taxes as citizens we implicitly hope and to a certain extent trust our government to use these taxes for the purpose they announce. This is in particular true for climate taxes. If a government decides to raise climate taxes on meat consumption because of the climate gases that occur in livestock farming, then there is the clear expectation that it is not just a cover-up to fill holes in the state budget, but a policy based on an ethically justified policy. Lykkeskov and Gjerris in "The Moral Justification Behind a Climate Tax in Denmark" discuss this case and find support among others in John Stuart Mill's Harm principle.

We mentioned already that trust relates to responsibility as a close companion. It may also relate to an ethics of care. This is at least the point of the policy paper by Wickson, Preston, Binimelis, Herrero, Hartly, Wynberg and Wynne. If we care, we may take on responsibility. A caring person is perhaps also a more trustworthy person? The paper is based on a highly contextualized real world narrative about an organic maize producer, which is used to introduce a concrete framework to help identify and articulate critical concerns. This text also points to the fact that narratives can draw attention to particularity and context, encourage the consideration and assessment of alternatives and grant individuals the power to tell and control their own stories. 
Well, final answers we do not get. We know how trust is important and we have learned about misplaced trust. We also see how narratives are important builders of trust. Not all expert tales trigger trust, and sometimes rightly so. We would wish that at least simple facts are respected in all our tales and narratives, and that those who seek our trust share this respect. But we loose the basis for acting ethically, if we do not respect facts, try to score points with "alternative facts", or are no longer interested in the arguments which may make those right with whom we disagree. Those who are beyond facts do not deserve trust.

\section{References}

Meijboom, F.L. 2008. Problems of trust: A question of trustworthiness: An ethical inquiry of trust and trustworthiness in the context of the agricultural and food sector. Utrecht University: Doctoral dissertation.

Ruger, J. 2003. Toward a theory of a right to health: Capability and incompletely theorized agreements. Yale Joural Law Humanities 18(2):273-326.

Thompson, P.B. 2015. From field to fork: Food ethics for everyone. U.S.: Oxford University Press. 